Article

\title{
Doping Effect of Graphene Nanoplatelets on Electrical Insulation Properties of Polyethylene: From Macroscopic to Molecular Scale
}

\author{
Ziang Jing ${ }^{1}$, Changming Li ${ }^{1}$, Hong Zhao ${ }^{1}$, Guiling Zhang ${ }^{2, *}$ and Baozhong Han ${ }^{1,3}$ \\ 1 Key Laboratory of Engineering Dielectric and its Application, Ministry of Education, \\ Harbin University of Science and Technology, Harbin 150040, China; hljjza@163.com (Z.J.); \\ hustlichangming@163.com (C.L.); hongzhao@hrbust.edu.cn (H.Z.); hbzhlj@163.com (B.H.) \\ 2 College of Chemical and Environmental Engineering, Harbin University of Science and Technology, \\ Harbin 150040, China \\ 3 Shanghai Qifan Wire and Cable Co., Ltd., Shanghai 200008, China \\ * Correspondence: guiling-002@163.com; Tel.: +86-541-8639-1657
}

Academic Editor: Der-Jang Liaw

Received: 20 April 2016; Accepted: 6 August 2016; Published: 10 August 2016

\begin{abstract}
The doping effect of graphene nanoplatelets (GNPs) on electrical insulation properties of polyethylene (PE) was studied by combining experimental and theoretical methods. The electric conduction properties and trap characteristics were tested for pure PE and PE/GNPs composites by using a direct measurement method and a thermal stimulated current (TSC) method. It was found that doping smaller GNPs is more beneficial to decrease the conductivity of PE/GNPs. The PE/GNPs composite with smaller size GNPs mainly introduces deep energy traps, while with increasing GNPs size, besides deep energy traps, shallow energy traps are also introduced. These results were also confirmed by density functional theory (DFT) and the non-equilibrium Green's function (NEGF) method calculations. Therefore, doping small size GNPs is favorable for trapping charge carriers and enhancing insulation ability, which is suggested as an effective strategy in exploring powerful insulation materials.
\end{abstract}

Keywords: graphene nanoplatelets doping effect; insulation property; polyethylene

\section{Introduction}

Since the 1950s, polyethylene (PE) has been the focused insulation material due to its outstanding electrical insulation property, excellent chemical stability, good processing formation performance, as well as efficient economic benefits. PE has been widely used in power apparatus as the main insulation material (e.g., capacitors, cables) [1,2]. Currently, much effort has been devoted to enhance the electric strength and the insulation property of PE under a direct current field by using various techniques [3-8]. Particularly, using alien fillers such as ceramic oxides (e.g., $\mathrm{Al}_{2} \mathrm{O}_{3}, \mathrm{ZnO}, \mathrm{SiO}_{2}$, etc.) to tailor the properties of PE has become a popular and effective strategy to develop insulation dielectrics with a specific high performance [9-13].

Many experimental works have found that doping of alien fillers could greatly improve the dielectric properties of PE composite and maintain high thermal endurance [14-19]. Fleming and his coworkers found that the conductivity of PE matrix composite containing $10 \mathrm{wt} \% \mathrm{TiO}_{2}$ decreased by 1-3 orders relative to undoped PE [20]. Yang et al. reported that the direct current conductivity of $\mathrm{PE} / \mathrm{SiO}_{2}$ decreased by 1-2 orders relative to pure PE [21]. Tian et al. stated that doping $\mathrm{ZnO}$ could introduce large amounts of deep trapping states and result in a decreasing conduction current of the $\mathrm{PE} / \mathrm{ZnO}$ composite [22]. Ishimoto et al. found that the conductivity of $\mathrm{PE} / \mathrm{MgO}$ composite was decreased compared with pure PE, irrespective of the size of $\mathrm{MgO}$ [23]. In prior works, $\mathrm{PE} /$ graphene 
with low content of graphene was generally used for electromagnetic shielding and anti-static electricity materials [24-26]. In this work, we found that graphene nanoplatelets (GNPs) are another good candidate for doping PE which can evidently lower the conductivity of the PE/GNPs composite. To the best of our knowledge, this is the first contribution regarding the doping effect of GNPs on the insulation properties of PE/GNPs composites.

Some theoretical works have been devoted to investigating the micro process and physical nature of the electrical phenomenon of the PE matrix composite. Montanari et al. calculated the electronic structure of PE and suggested a $6.0 \mathrm{eV}$ forbidden band gap [27]. Righi et al. studied the electronic structure of the PE surface by considering an orthorhombic crystalline PE slab and the electron affinity was calculated to be -0.10 and $-0.17 \mathrm{eV}$ for the (001) and (110) surface, respectively [28]. Huzayyin and his coworkers used density functional theory (DFT) to study the effect of various chemical impurities in PE on the electronic structures, trap depths, electron densities, and inter-chain interactions. They stated that both shallow traps and deep traps could be introduced into PE by impurities [29]. Based on DFT and ab initio calculations, Meunier et al. validated that physical and chemical defects could both trap electrons [30-32]. To the best of our knowledge, no theoretical works have been related to GNPs doping effect on the dielectric property of PE.

In this work, the doping effect of GNPs on the dielectric property of PE was investigated by combining experimental and theoretical methods. The conduction properties and trap characteristics were tested for pure PE and PE/GNPs composites firstly by using a direct measurement method and a thermal stimulated current (TSC) method. Then, the trap-limited band conduction properties of PE and PE/GNPs composites were studied using DFT and Non-Equilibrium Green Formula (NEGF) methods. The theoretical analysis qualitatively correlates well with the results from the experiment.

\section{Experimental Works}

\subsection{Samples Preparation}

Low density PE (LDPE) was used in this study, which was produced by SINOPEC Beijing Yanshan Petrochemical Co., Ltd., Beijing, China. The GNPs were manufactured by XG Sciences, Inc., Lansing, MI, USA. Two kinds of GNPs with different sizes (denoted as GNP(1) and GNP(2), respectively) were selected for fabricating PE/GNPs composites. The maximum diameters of flake plane of GNP(1) and GNP(2) were about 1.0 and $0.65 \mu \mathrm{m}$, respectively, which were obtained through a proprietary manufacturing process by the manufacturer [33]. The content of both kinds of GNPs was $1.0 \mathrm{wt} \%$ and the thickness was both $30-40 \mathrm{~nm}$. Therefore, GNP(2) has more flakes than GNP(1) at the same quality. The PE/GNPs composite was prepared by a melt blending method using a torque rheometer. The LDPE was first melted in the torque rheometer for $5 \mathrm{~min}$ at $383 \mathrm{~K}$ with the speed of the rotors at $50 \mathrm{rpm}$, and then the GNP(1) or GNP(2) was filled into the LDPE. The GNPs and PE were mixed for $20 \mathrm{~min}$ at $393 \mathrm{~K}$ with the speed of the rotors at $70 \mathrm{rpm}$. After that, both the pure PE and the two kinds of PE/GNPs composites were pressed into thin films under a plate vulcanizer at $383 \mathrm{~K}$, with thicknesses of either 80 or $200 \mu \mathrm{m}$, the thicker films being used in the conduction characteristic test and the thinner films in the TSC test. All samples were evaporated with an Al electrode on both sides with a diameter of $25 \mathrm{~mm}$.

\subsection{Conduction Characteristic Test}

Based on the direct measurement method, the conduction properties of pure PE, PE/GNP(1) composite, and PE/GNP(2) composite were tested. Quasi steady state current was obtained by the electrometer, Keithley6517B. The measurement was carried out at room temperature. Step voltages from 1000 to $10,000 \mathrm{~V}$ were applied on the samples with $200 \mu \mathrm{m}$ thickness. The interval of the step voltages was $1000 \mathrm{~V}$ and the sampling time was $600 \mathrm{~s}$. For ensuring accuracy of the experimental results, the measurement was repeated four times under every testing voltage. The final result was the 
average value of the four measurements at every testing voltage. The obtained conductivity-electric field $(\Upsilon-E)$ characteristic curves are shown in Figure 1.

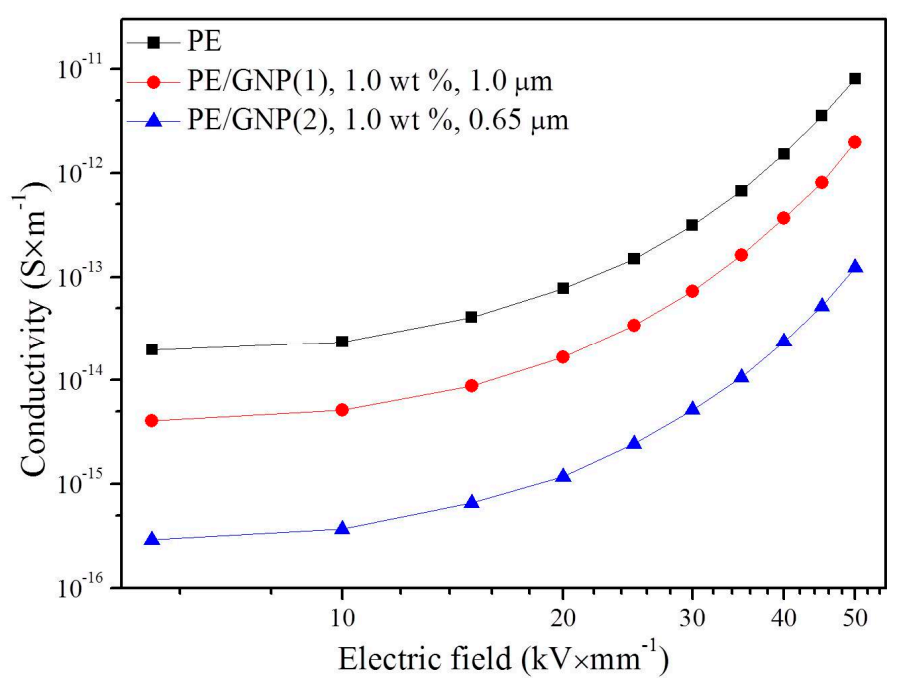

Figure 1. $\Upsilon-E$ characteristic curves of pure PE, PE/GNP(1), $1.0 \mathrm{wt} \%, 1.0 \mu \mathrm{m}$ composite, and $\mathrm{PE} / \mathrm{GNP}(2), 1.0 \mathrm{wt} \%, 0.65 \mu \mathrm{m}$ composite (PE and GNP refer to polyethylene and graphene nanoplatelet, respectively).

\subsection{Electron Trap Characteristic Test}

The electron trap characteristic was tested based on the TSC method. The TSC method is a significant method to study important parameters of electrets such as thermal charge mobility and trapped charge density, etc. For example, using the TSC method, He et al. obtained the trap distribution of $\mathrm{PE} / \mathrm{MgO}$, Tian et al. discussed the trap property of $\mathrm{PE} / \mathrm{ZnO}$, and Han et al. investigated the trap distribution of PE/zeolite [34-36]. In this work, the sample was firstly charged under dc electric field $(40 \mathrm{kV} / \mathrm{mm}$ ) at $323 \mathrm{~K}$. After charging $30 \mathrm{~min}$, the sample was rapidly cooled to $270 \mathrm{~K}$, and then shorted until the current decayed to below $2 \mathrm{pA}$ for eliminating the influence of stray current and interfacial charge. Then, the sample was heated to $370 \mathrm{~K}$ at aspeed of $2 \mathrm{~K} / \mathrm{min}$. The change of depolarization current versus the temperature was recorded by Labview software and the result is shown in Figure 2a.

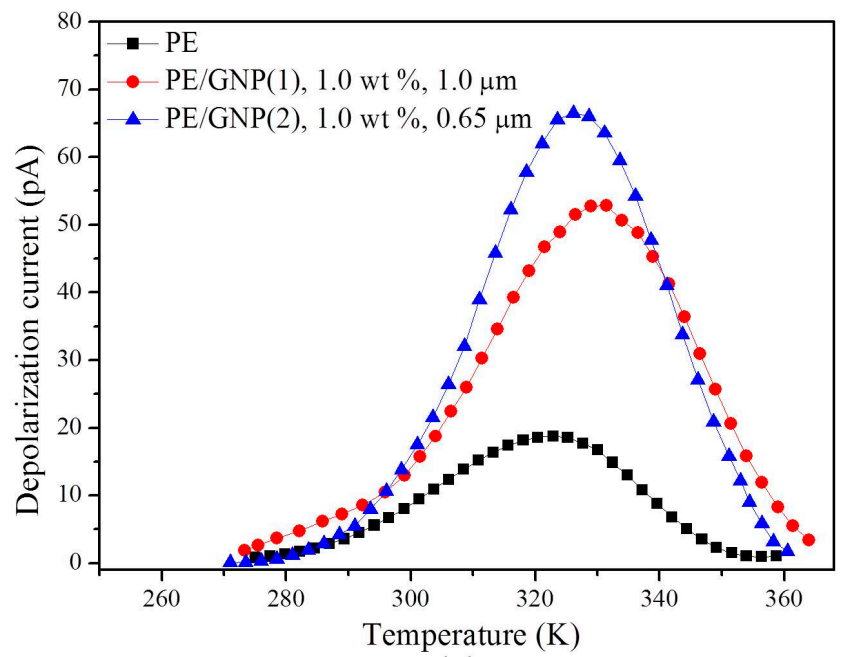

(a)

Figure 2. Cont. 


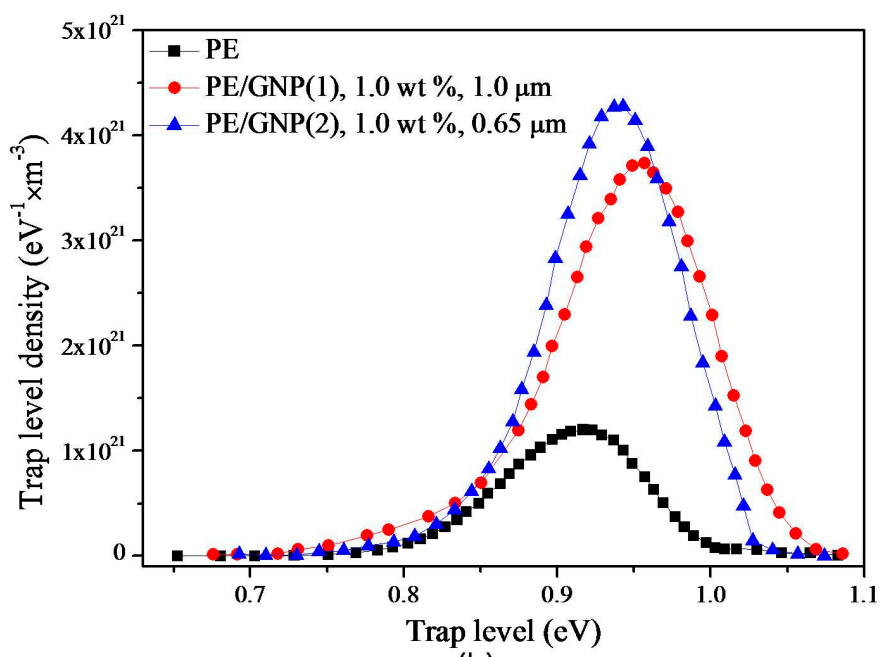

(b)

Figure 2. (a) Depolarization current versus temperature of pure PE, PE/GNP(1), $1.0 \mathrm{wt} \%, 1.0 \mu \mathrm{m}$ composite, and PE/GNP(2), 1.0 wt \%, $0.65 \mu \mathrm{m}$ composite; (b) Trap level distribution of pure PE, PE/GNP(1), $1.0 \mathrm{wt} \%, 1.0 \mu \mathrm{m}$ composite, and PE/GNP(2), $1.0 \mathrm{wt} \%, 0.65 \mu \mathrm{m}$ composite.

\subsection{Results and Discussion}

Figure 1 shows the $\Upsilon$ - $E$ characteristic curves of pure PE and GNPs/PE composites. Clearly, adding GNPs into PE could markedly reduce the conductivity on comparing with pure PE. The conductivity of PE/GNP(1) composite is about one order of magnitude lower than that of pure PE. Particularly, PE/GNP(2) composite exhibits lower conductivity than PE/GNP(1) composite. This means that doping smaller GNPs flack is more beneficial in decreasing the conductivity of PE/GNPs. Such a case is also confirmed by theoretical prediction as shown in the following section.

The depolarization currents versus temperature for the thermal activation for pure PE, PE/GNP(1), and PE/GNP(2) are plotted in Figure 2a. The voltage was applied for $30 \mathrm{~min}$ so that the space charge could be well accumulated in the PE or PE/GNPs composites. The depolarization current and the temperature are important parameters in investigating charge carrier trap characteristics. Generally, the deeper the trap, the higher is the trap energy, that is, a higher temperature is needed for detrapping of the trapped charge. If ignoring retrapping of the detrapped charge and the recombination of different polar carriers, all the detrapping charges contribute to the depolarization current. The relationship between the depolarization current $I$, the trap energy $E$, and the temperature $T$ are given as Equation (1) [37].

$$
J(T)=\frac{I(T)}{S}=\frac{e l^{2}}{2 d} \int_{E_{v}}^{E_{c}} f_{0}(E) N_{t}(E) e_{n} e^{-\frac{1}{\beta} \int_{T_{0}}^{T} e_{n} \mathrm{~d} T} \mathrm{~d} E
$$

where

$$
e_{n}=v \exp \left(-\frac{E_{t}}{k T}\right)
$$

$J(T)$ is the current density, $S$ is the area of the electrode, $e$ is electronic charge quantity, $f_{0}$ is an equation referring to the initial occupancy of a trap level, $N_{t}$ is the trap level density of the localized states, $E$ is the trap energy, $e_{n}$ is the rate of emission of electrons from a level at energy $E$ and temperature $T, v$ is the escape frequency of trapped electrons, $k$ is the Boltzmann constant, $d$ is the thickness of the film, $l$ is the penetration depth of the injected electrons, and $\beta$ is the heating rate. 
According to the numerical calculation method addressed by Tian et al. [38], Equation (1) can be simplified as Equation (3).

$$
\begin{aligned}
J(t) & =\frac{e l^{2}}{2 d} f_{0}\left(E_{m}\right) N_{t}\left(E_{m}\right) \frac{d E_{m}}{d t} \\
& =\frac{e l^{2}}{2 d} f_{0}\left(E_{m}\right) N_{t}\left(E_{m}\right) \frac{k T}{t}
\end{aligned}
$$

where

$$
\begin{gathered}
t=\frac{T-T_{0}}{\beta} \\
E_{m}=k T \ln \left[\frac{v\left(T-T_{0}\right)}{\beta}\right]
\end{gathered}
$$

$E_{\mathrm{m}}$ is also defined as the demarcation energy [38].

By combining Equations (3) and (4), Equation (3) can be converted to Equation (6).

$$
f_{0} N_{t}\left(E_{m}\right)=\frac{2 d\left(T-T_{0}\right)}{e l^{2} \beta k T} J(T)
$$

Assuming all the traps were fully filled and $f_{0}=1$, by combining Equations (5) and (6), the trap level density of pure PE, PE/GNP(1), and PE/GNP(2) can be obtained as plotted in Figure $2 b$. The trap energy levels for both PE and PE/GNPs composites are in the range of $0.70-1.10 \mathrm{eV}$. The trap level density peak of pure PE is around $0.92 \mathrm{eV}$, consistent with the result of $0.92 \mathrm{eV}$ obtained by Ieda et al. [39]. Evidently, the peak of the trap level density shifts to higher energy level after introducing the GNPs dopants. The density peaks of PE/GNP(1) composite and PE/GNP(2) were around $0.95 \mathrm{eV}$ and $0.93 \mathrm{eV}$, respectively. At a lower energy level $(<0.86 \mathrm{eV})$, the trap density of $\mathrm{PE} / \mathrm{GNP}(1)$ is larger than PE/GNP(2). And at a higher energy level $(>0.98 \mathrm{eV})$, the trap density of $\mathrm{PE} / \mathrm{GNP}(1)$ is larger than PE/GNP(2). This means that PE/GNP(2) with a smaller size GNP(2) mainly introduces deep energy traps while PE/GNP(1) with a larger size GNP(1) introduces not only deep energy traps but also shallow energy traps. The effectiveness of the deep traps and shallow traps on the process of carrier transport is different [40]. The shallow trap states are in thermal equilibrium with valence band $(\mathrm{VB})$ or conduction band $(\mathrm{CB})$ : transport can occur by thermal activation of carriers to $\mathrm{VB}$ or $\mathrm{CB}$, leading to the concept of conduction through multiple trapping/detrapping steps, which is beneficial to enhancing the charge transport. However, the deep trap states are far away from VB or $\mathrm{CB}$, and this is thought to control the space charge and hinder the charge transport. Therefore, small size GNPs are beneficial in trapping charge carriers and enhancing insulation ability. In order to further illustrate the energy level changes with the introduction of GNPs, the volume average trap densities of the samples can be estimated through the integral of the trap level density curves. The volume average trap densities of pure PE, PE/GNP(1) composite, and PE/GNP(2) composite, are $1.38 \times 10^{20} \mathrm{~m}^{-3}, 4.54 \times 10^{20} \mathrm{~m}^{-3}$, and $4.98 \times 10^{20} \mathrm{~m}^{-3}$, respectively. $\mathrm{PE} / \mathrm{GNP}(2)$ has higher volume trap densities than PE/GNP(1) owing to the fact that PE/GNP(1) has a larger amount of molecular segments than PE/GNP(2).

\section{Theoretical Works}

In order to qualitatively investigate the doping effect of GNPs on the dielectric property of PE/GNPs composite, we constructed calculation models by assuming graphene flacks were added into PE, as shown in Figure 3. The electronic structures and transport properties of PE and PE/GNPs were analyzed by combining the calculated results from the DFT and NEGF methods. 


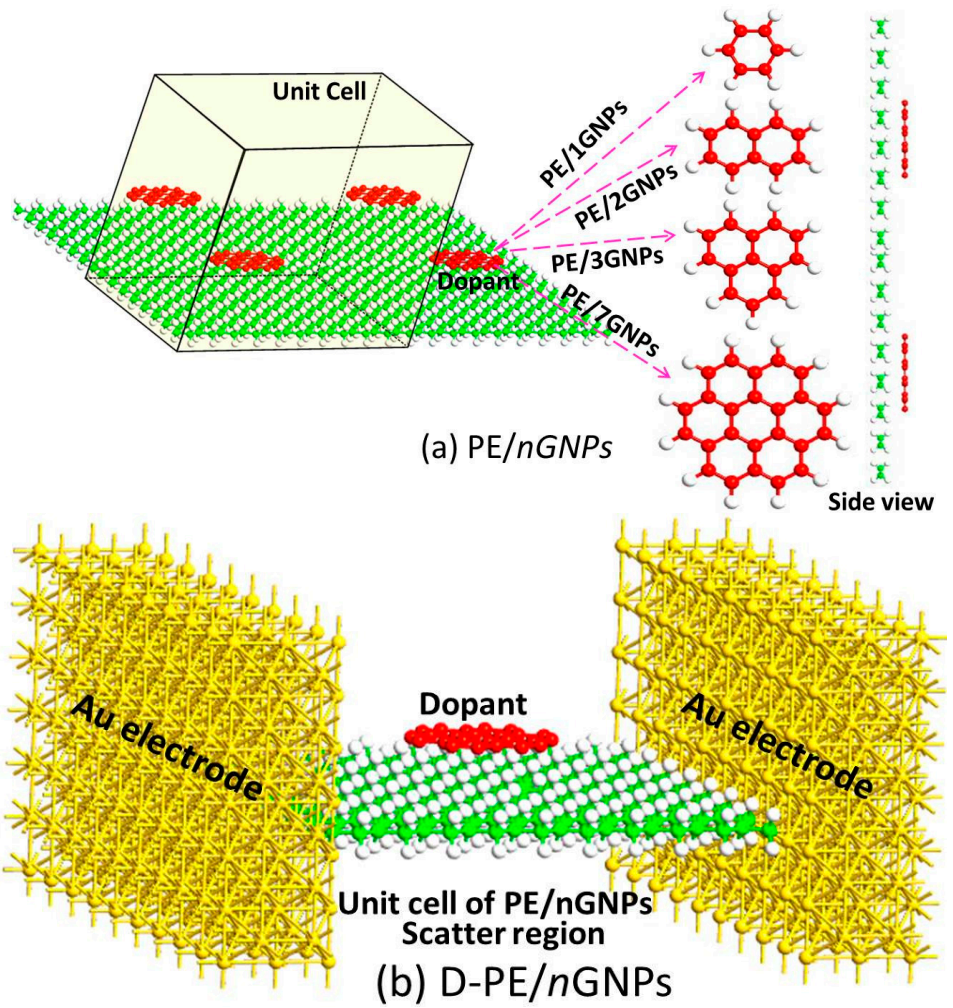

Figure 3. (a) Optimized structures of PE/ $n$ GNPs $(n=1,2,3,7)$. The unit cell for calculations is also given; (b) Two-probe devices of PE/ $n$ GNPs $(n=1,2,3,7)$ as one supercell were sandwiched between two Au (100) $-(7 \times 9)$ electrodes, five layers were used for the left and right Au electrodes.

\subsection{Electronic Structure Calculation}

For computing electronic structures, the infinite system of pure PE and PE/GNPs was modeled using a periodic condition in the plane of YZ. Four models were chosen here, denoted as PE $/ n \mathrm{GNPs}$, $n=1,2,3$, and 7 , meaning one-ring graphene, two-ring graphene, three-ring graphene, and seven-ring graphene were introduced into one unit cell of PE, respectively (Figure 3a). Each unit cell contained eight PE chains and each PE chain consisted of twenty $C$ atoms. These eight PE chains were parallel arrayed along the $\mathrm{Y}$-axis direction. The longitudinal repeat direction of the PE chain was along the Z-axis. The graphene plane was also in the $\mathrm{YZ}$ plane and superposed upon the $8 \mathrm{PE}$ plane. All the periodic systems were fully optimized until the maximum absolute force was less than $0.02 \mathrm{eV} / \AA$. Computations for the infinitely long systems were performed using an ab initio code package, Atomistix ToolKit (ATK), which is based on combination of DFT and NEGF methods [41-44]. A generalized gradient approximation (GGA) within the Perdew-Burke-Ernzerhof (PBE) formalism was employed to describe the exchange correlations between electrons. A double- $\zeta$ basis functional with polarization (DZP) was used for all atoms. The k-point was set as $(1 \times 50 \times 50)$ in the Brillouin zone $(x, y, z$ directions, respectively).

\subsection{Electronic Structure}

Figure 4 plots the band structures and average projected density of states (PDOS) of PE, PE/1GNPs, PE/2GNPs, PE/3GNPs, and PE/7GNPs, as well as the Kohn-Sham orbitals near the Fermi level $\left(E_{\mathrm{f}}\right)$. The distance between the PE and the graphene is around $3.0 \AA$, suggesting a Van der Waal's interaction. For pure PE, the forbidden band spans a large band gap of about $6.2 \mathrm{eV}$, indicating typical insulation character. This is in agreement with previous experimental observations and DFT calculations [27]. In pure PE, the Kohn-Sham orbitals of VB and CB seem to cover the whole of the 
main chain, and thereby, the electron and hole in $\mathrm{CB}$ and VB are freely mobile and show no localized level. The PE VB state spreads mainly along the longitation chains, while the CB state extends not only to the longitation chains, but also overlaps between the inter-chains [40]. Such inter-chain character for $\mathrm{CB}$ and the intra-chain character for VB were also confirmed by Serra [45]. Therefore, carrier transport in PE may proceed via two transport pathways: one is named direct intra-chain mechanism whose carriers can transport along a PE chain; another is named indirect inter-chain mechanism whose carriers can transport by hopping to a neighboring PE chain.
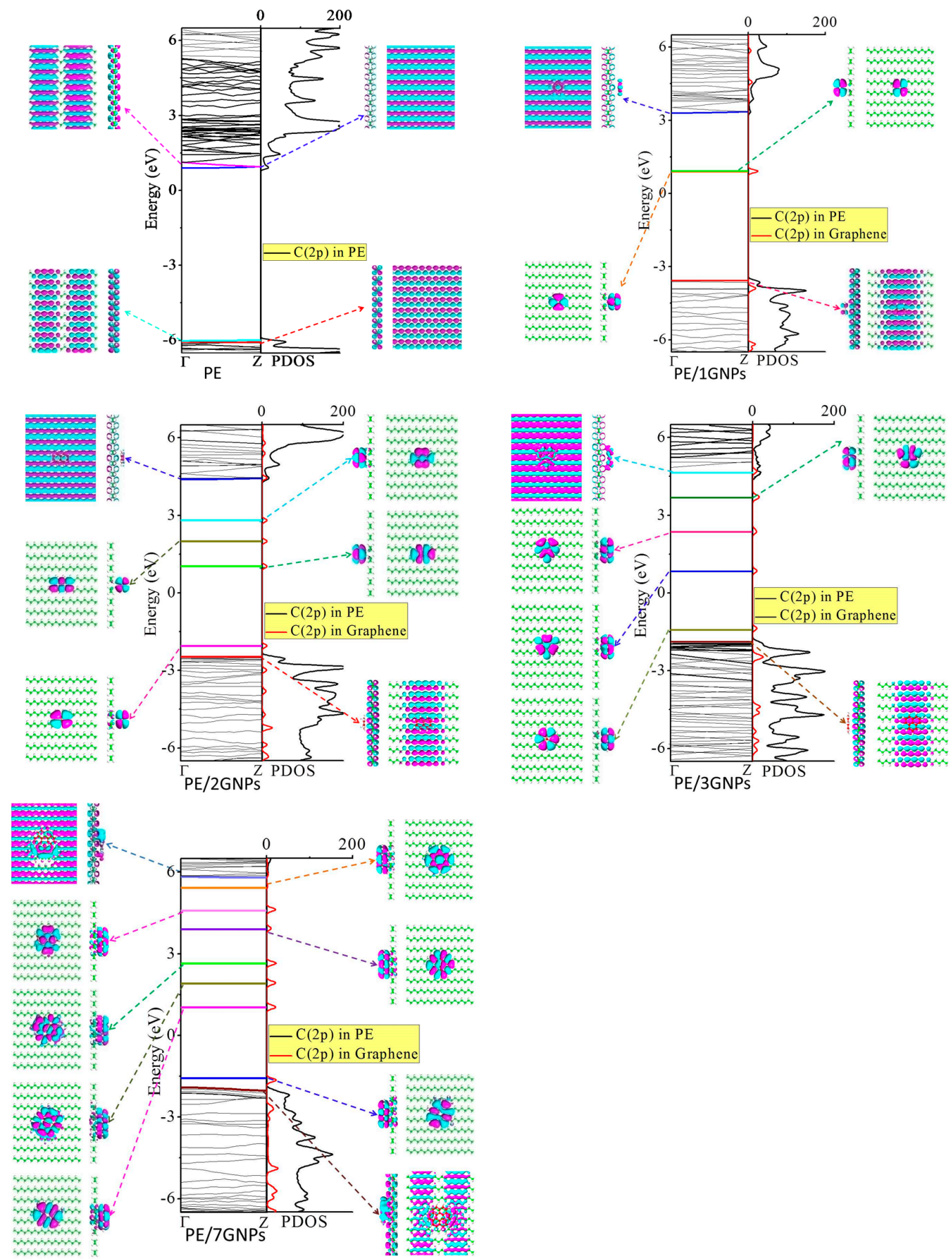

Figure 4. Calculated band structures and PDOS of PE, PE/1GNPs, PE/2GNPs, PE/3GNPs, and $\mathrm{PE} / 7 \mathrm{GNPs}$ and the Kohn-Sham orbitals corresponding to the energy levels (highlighted in color lines) near $E_{\mathrm{f}}$ at the $\Gamma$ point. The iso-surface value is $0.005 \mathrm{eV}$. 
When graphene pieces are introduced into PE, localized impurity bands originating from the graphene segment appear in the forbidden band region. As the size of the graphene plane increases, the amount of the impurity states in the forbidden band increases. Most of them are localized above the $E_{\mathrm{f}}$, serving as electron trap states. Based on the difference between the trap energy level and $E_{\mathrm{f}}$, the traps are divided into deep traps and shallow traps. The trap depth for the electron can be evaluated as the difference between the unoccupied impurity band nearest $\mathrm{CB}$ and $\mathrm{CB}$. The trap depth for the hole is also calculated as the difference between the occupied impurity band nearest VB and the VB. The calculated electron trap depths for PE/1GNPs, PE/2GNPs, PE/3GNPs, and PE/7GNPs are 2.2, $1.7,0.6$, and $0.3 \mathrm{eV}$, respectively. The calculated hole trap depths for PE/nGNPs are of about $0.0-0.4 \mathrm{eV}$, acting as shallow traps. For PE/1GNPs, two degenerate graphene bands locate just above the $E_{\mathrm{f}}$ with a large trap depth, serving as deep trap states for localized electrons. On enlarging the graphene segment, more shallow trap and deep trap states are introduced below the CB. Especially, in PE/7GNPs, a series of quasi-continuous graphene bands are formed in the forbidden band region of PE. On the other hand, the hole trap state is also introduced above the VB in PE/2GNPs, PE/3GNPs, and PE/7GNPs. Moreover, adding graphene segments also induces upshift of VB, and this upshifting becomes larger with the increasing size of graphene. This means that adding a large graphene segment is prone to electron ionization from PE VB into the trap state. In other words, in the PE/GNPs composite with large graphene segments, electrons could easily fall into graphene species, transfer within the graphene itself, and then be detrapped to the CB of PE. The trap depths for the electron are decreased with increasing size of the graphene segment. Therefore, introducing graphene into PE has twofold opposite effects. One is constructive to the insulator property of PE, especially for small graphene segments; the other is deconstructive to the insulator property of PE, especially for large graphene segments.

\subsection{Transport Property Calculation}

For computing transport properties, we carved out one unit cell of PE, PE/1GNPs, PE/2GNPs, PE/3GNPs, and PE/7GNPs as the central scatter region based on the optimized periodic structures to be sandwiched between two Au electrodes (Figure 3b). The unit cell was long enough $(\sim 25 \AA)$ to neglect the interaction between the left and right electrodes. The semi-infinite Au electrodes were modeled by two $\mathrm{Au}(100)-(7 \times 9)$ surfaces, and five layers were used for the left and right side. Calculations were carried out by changing the applied bias in the step of $0.2 \mathrm{~V}$ in the range of $-1.0 \sim 1.0 \mathrm{~V}$. The five two-probe devices were denoted as D-PE, D-PE/1GNPs, D-PE/2GNPs, D-PE/3GNPs, and D-PE/7GNPs, respectively. Computations were performed using ATK package. A generalized gradient approximation (GGA) within the Perdew-Burke-Ernzerhof (PBE) formalism is employed to describe the exchange correlations between electrons. A double-æ basis functional with polarization (DZP) was used for all atoms. The $\mathrm{k}$-point was set as $(1 \times 50 \times 50)$ in Brillouin zone $(x, y, z$ directions, respectively).

\subsection{Transport Property}

The calculated $I-V$ curves are shown in Figure 5. From Figure 5, the conduction current in D-PE/1GNPs and D-PE/2GNPs is decreased compared to the pure PE under certain external bias voltages. However, with increasing graphene flack size, the conduction current of the doped PE matrix tends to increase. When the seven-ring graphene flacks are filled into PE, the conduction current is even larger than the pure PE. Usually, the conduction current is relevant to the transmission peak at the $E_{\mathrm{f}}$ under free bias. Figure 6 gives the transmission spectrum (TS) at $0.0 \mathrm{~V}$. Clearly, the values of TS at $E_{\mathrm{f}}$ $\left(\mathrm{T}_{0}\right)$ follow the sequence of D-PE/1GNPs $<$ D-PE/2GNPs $<$ D-PE/3GNPs $\approx$ D-PE $<$ D-PE/7GNPs. This result is consistent with the $I-V$ character in Figure 5. Two reasons may contribute to the high conduction current of D-PE/7GNPs. One is that trapped carries could be easily detrapped from the shallow trap levels of graphene to the PE. Another is that the seven-ring graphene flack is large enough in the scatter region to serve as a transport channel directly. 


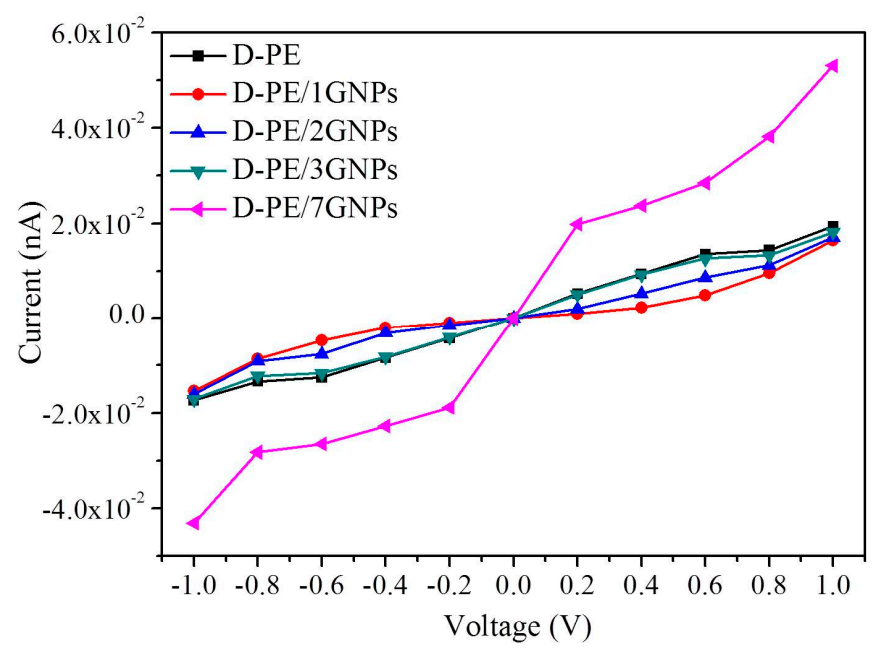

Figure 5. Computed $I-V$ curves of D-PE, D-PE/1GNPs, D-PE/2GNPs, D-PE/3GNPs, and D-PE/7GNPs two-probe devices.
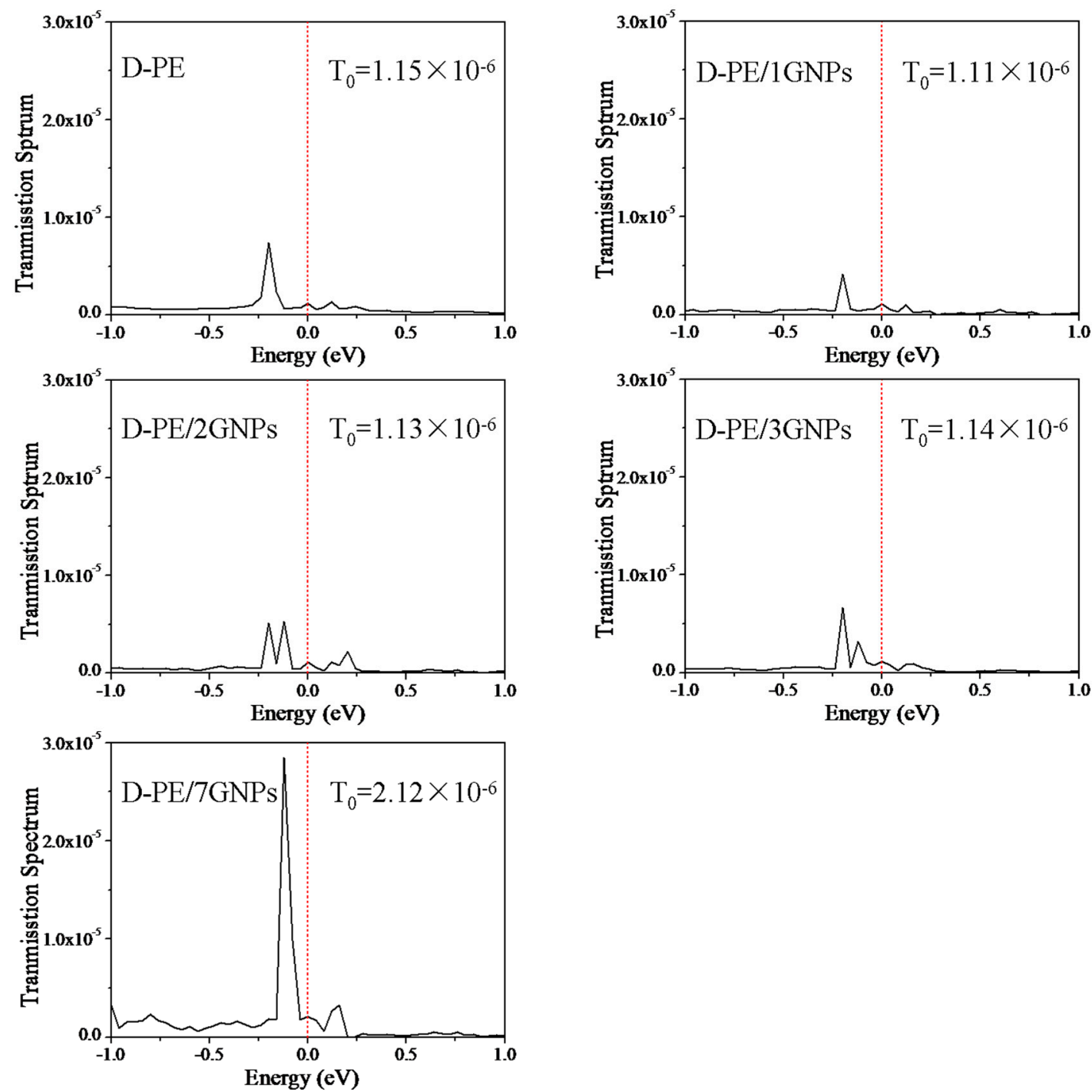

Figure 6. Transmission spectra of D-PE, D-PE/1GNPs, D-PE/2GNPs, D-PE/3GNPs, and D-PE/7GNPs two-probe devices at $0.0 \mathrm{~V}$ bias voltages. 
From the theoretical conclusion based on the PE/GNPs composite, we can deduce that adding appreciable amount of GNPs of a small size into PE is favorable for enhancing the insulation property of PE. This is in agreement with the experimental result as mentioned above.

\section{Conclusions}

The doping effect of GNPs on the dielectric properties of PE was studied by combining experimental and theoretical methods. The conduction properties and trap characteristics were tested for pure PE and PE/GNPs composite by using a direct measurement method and the TSC method. It was found that doping smaller GNPs is more beneficial to decrease the conductivity of PE. The PE/GNPs composite with smaller size GNPs mainly introduces deep energy traps, while with increasing GNPs size, besides deep energy traps, shallow energy traps are also introduced. These results are also confirmed from the band structures and $I-V$ curves of a series of PE/GNPs composites obtained from DFT and NEGF calculations. Therefore, doping small size GNPs is beneficial to trapping charge carriers and enhancing insulation ability, which is suggested as an effective strategy to explore powerful insulation materials.

Acknowledgments: G.L.Z. is supported by the National Science Foundation of China (grant No. 51473042). B.Z.H. is supported by the National Science Foundation of China (grant No. 51337002) and Natural Science Foundation for Distinguished Young Scholars of Heilongjiang Provence (JC201409).

Author Contributions: H.Z. and C.L. conceived and designed the experiments; Z.J. performed the experiments; Z.J. and G.Z. analyzed the data; B.H. contributed reagents/materials/analysis tools; Z.J. wrote the paper.

Conflicts of Interest: The authors declare no conflict of interest.

\section{References}

1. Teyssedre, G.; Laurent, C. Advances in high-field insulating polymeric materials over the past 50 years. IEEE Electr. Insul. Mag. 2013, 29, 26-36. [CrossRef]

2. Teyssedre, G.; Laurent, C.; Montanari, G.C.; Campus, A.; Nilsson, U.H. From LDPE to XLPE: Investigating the change of electrical properties. Part II. Luminescence. IEEE. Trans. Dielectr. Electr. Insul. 2005, 12, 447-454. [CrossRef]

3. Iida, K.; Kim, J.S.; Nakamura, S.; Sawa, G. Effects of molecular structure on electrical conduction in low-density polyethylene above its melting point. IEEE Trans. Electr. Insul. 1992, 27, 391-398. [CrossRef]

4. Suh, K.S.; Lee, C.R.; Noh, J.S.; Tanaka, J.; Damon, D.H. Electrical conduction in polyethylene with semiconductive electrodes. IEEE Trans. Dielectr. Electr. Insul. 1994, 1, 224-230. [CrossRef]

5. Pelissou, S.; St-Onge, H.; Wertheimer, M.R. Electrical conduction of polyethylene below and above its melting point. IEEE Trans. Dielectr. Electr. Insul. 1988, 23, 325-333. [CrossRef]

6. Hosier, I.L.; Vaughan, A.S.; Swingler, S.G. Structure-property relationships in polyethylene blends: The effect of morphology on electrical breakdown strength. J. Mater. Sci. 1997, 32, 4523-4531. [CrossRef]

7. Perlman, M.; Kumar, A.; Coelho, R.; Aladenize, B. Steady-state conduction in linear low-density polyethylene with Poole-lowered trap depth. IEEE Trans. Electr. Insul. 1990, 25, 419-425. [CrossRef]

8. Zhou, T.; Chen, G.; Liao, R.; Xu, Z. Charge trapping and detrapping in polymeric materials: Trapping parameters. J. Appl. Phys. 2011, 110, 3626468. [CrossRef]

9. Wang, S.J.; Zha, J.W.; Li, W.K.; Dang, Z.M. Distinctive electrical properties in sandwich-structured $\mathrm{Al}_{2} \mathrm{O}_{3} /$ low density polyethylene nanocomposites. Appl. Phys. Lett. 2016, 108, 031605. [CrossRef]

10. Tian, F.; Lei, Q.; Wang, X.; Wang, Y. Investigation of electrical properties of LDPE/ZnO nanocomposite dielectrics. IEEE Trans. Dielectr. Electr. Insul. 2012, 19, 763-769. [CrossRef]

11. Chen, J.; Yin, Y.; Li, Z.; Xiao, D.M. Electrical prestressing of high-electric-field conduction in composite of low-density polyethylene/nano-SiO ${ }_{x}$. Jpn. J. Appl. Phys. 2005, 44, 940-947. [CrossRef]

12. Chodak, I.; Krupa, I. "Percolation effect" and mechanical behavior of carbon black filled polyethylene. J. Mater. Sci. Lett. 1999, 18, 1457-1459. [CrossRef]

13. Kalaitzidou, K.; Fukushima, H.; Drzal, L.T. A Route for Polymer Nanocomposites with Engineered Electrical Conductivity and Percolation Threshold. Materials 2010, 3, 1089-1103. [CrossRef] 
14. Huang, X.; Jiang, P.; Xie, L. Ferroelectric polymer/silver nanocomposites with high dielectric constant and high thermal conductivity. Appl. Phys. Lett. 2009, 95, 242901. [CrossRef]

15. Chen, G.; Fu, M.; Liu, X.Z.; Zhong, L.S. Ac aging and space-charge characteristics in low-density polyethylene polymeric insulation. J. Appl. Phys. 2005, 97, 083713. [CrossRef]

16. Li, S.T.; Zhao, N.; Nie, Y.; Wang, X.; Chen, G. Space charge characteristics of LDPE nanocomposite/LDPE insulation system. IEEE Trans. Dielectr. Electr. Insul. 2015, 22, 92-100. [CrossRef]

17. Montanari, G.C.; Mazzanti, G.; Palmieri, F.; Motori, A.; Perego, G. Space-charge trapping and conduction in LDPE, HDPE and XLPE. J. Phys. D Appl. Phys. 2001, 34, 2902-2911. [CrossRef]

18. An, Z.; Liu, C.; Chen, X.; Zheng, F.; Zhang, Y. Correlation between space charge accumulation in polyethylene and its fluorinated surface layer characteristics. J. Phys. D Appl. Phys. 2011, 45, 35302. [CrossRef]

19. Tanaka, Y.; Li, Y.; Takada, T.; Ikeda, M. Space charge distribution in low-density polyethylene with charge-injection suppression layers. J. Phys. D Appl. Phys. 1998, 28, 1232-1238. [CrossRef]

20. Fleming, R.J.; Pawlowski, T.; Ammala, A.; Casey, P.S. Electrical conductivity and space charge in LDPE containing $\mathrm{TiO}_{2}$ nanoparticles. IEEE Trans. Dielectr. Electr. Insul. 2005, 12, 745-753. [CrossRef]

21. Yang, J.; Liu, C.; Zheng, C.; Zhao, H.; Wang, X.; Gao, M. Effects of interfacial charge on the DC dielectric properties of nanocomposites. J. Nanomater. 2016, 5, 1-11. [CrossRef]

22. Tian, F.; Lei, Q.; Wang, X.; Wang, Y. Effect of deep trapping states on space charge suppression in polyethylene/ZnO nanocomposite. Appl. Phys. Lett. 2011, 99, 142903. [CrossRef]

23. Ishimoto, K.; Kanegae, E.; Ohki, Y.; Tanaka, T.; Sekiguchi, Y. Superiority of dielectric properties of LDPE/MgO nanocomposites over microcomposites. IEEE Trans. Dielectr. Electr. Insul. 2009, 16, 1735-1742. [CrossRef]

24. Kuilla, T.; Bhadra, S.; Yao, D.; Nam, H.K.; Saswata, B.; Joong, H.L. Recent advances in graphene based polymer composites. Prog. Polym. Sci. 2010, 35, 1350-1375. [CrossRef]

25. Kim, H.; Kobayashi, S.; Abdurrahim, M.A.; Minglun, J.Z.; Albina, K.; Marc, A.H.; Ahmed, A.A.; Christopher, W.M. Graphene/polyethylene nanocomposites: Effect of polyethylene functionalization and blending methods. Polymer 2011, 52, 1837-1846. [CrossRef]

26. Kuila, T.; Bose, S.; Mishra, A.K. Effect of functionalized graphene on the physical properties of linear low density polyethylene nanocomposites. Polym. Test. 2012, 31, 31-38. [CrossRef]

27. Montanari, B.; Jones, R.O. Density functional study of crystalline polyethylene. Chem. Phys. Lett. 1997, 272, 347-352. [CrossRef]

28. Righi, M.C.; Scandolo, S.; Serra, S.; Larlori, S.; Tosatti, E. Surface states and negative electron affinity in polyethylene. Phys. Rev. Lett. 2001, 87, 076802. [CrossRef] [PubMed]

29. Huzayyin, A.; Boggs, S.; Ramprasad, R. Density functional analysis of chemical impurities in dielectric polyethylene. IEEE Trans. Dielectr. Electr. Insul. 2010, 17, 926-930. [CrossRef]

30. Meunier, M.; Quirke, N. Molecular modeling of electron trapping in polymer insulators. J. Chem. Phys. 2000, 113, 369-376. [CrossRef]

31. Meunier, M.; Quirke, N.; Aslanides, A. Molecular modeling of electron traps in polymer insulators: Chemical defects and impurities. J. Chem. Phys. 2001, 115, 2876-2881. [CrossRef]

32. Anta, J.A.; Marcelli, G.; Meunier, M.; Quirke, N. Models of electron trapping and transport in polyethylene: Current-voltage characteristics. J. Appl. Phys. 2002, 92, 1002-1008. [CrossRef]

33. XG Sciences, USA. Available online: www.xgsciences.us/index.html (accessed on 18 April 2016).

34. Peng, S.; He, J.; Hu, J.; Huang, X.; Jiang, P. Influence of functionalized MgO nanoparticles on electrical properties of polyethylene nanocomposites. IEEE. Trans. Dielectr. Electr. Insul. 2015, 22, 1512-1519. [CrossRef]

35. Tian, F.; Yao, J.; Li, P.; Wang, Y.; Wu, M.; Lei, Q. Stepwise electric field induced charging current and its correlation with space charge formation in LDPE/ZnO nanocomposite. IEEE. Trans. Dielectr. Electr. Insul. 2015, 22, 1232-1239. [CrossRef]

36. Han, B.; Wang, X.; Sun, Z.; Yang, J.; Lei, Q. Space charge suppression induced by deep traps in polyethylene/zeolite nanocomposite. Appl. Phys. Lett. 2013, 102, 012902. [CrossRef]

37. Lei, Q.; Tian, F.; Yang, C.; He, L.; Wang, Y. Modified isothermal discharge current theory and its application in the determination of trap level distribution in polyimide films. J. Electrost. 2010, 68, 243-248.

38. Tian, F.; Bu, W.; Shi, L.; Yang, C.; Wang, Y. Theory of modified thermally stimulated current and direct determination of trap level distribution. J. Electrost. 2011, 69, 7-10. [CrossRef]

39. Ieda, M. Electrical conduction and carrier traps in polymeric materials. IEEE Trans. Dielectr. Electr. Insul. 1984, 19, 162-178. [CrossRef] 
40. Nelson, J.K. Dielectric Polymer Nanocomposites; Springer: New York, NY, USA, 2010.

41. Taylor, J.; Guo, H.; Wang, J. Ab Initio Modeling of Quantum Transport Properties of Molecular Electronic Devices. Phys. Rev. B Condens. Matter Mater. Phys. 2001, 63, 245407. [CrossRef]

42. Brandbyge, M.; Mozos, J.L.; Ordejón, P.; Taylor, J.; Stokbro, K. Density-Functional Method for Nonequilibrium Electron Transport. Phys. Rev. B Condens. Matter Mater. Phys. 2002, 65, 165401. [CrossRef]

43. Soler, J.M.; Artacho, E.; Gale, J.D.; Garcia, A.; Junquera, J.; Ordejon, P.; Sanchez-Portal, D. The SISTA method for Ab Initio Order-N Materials Simulation. J. Phys. Condens. Matter 2002, 14, 2745-2779. [CrossRef]

44. ATK, Version 13.8, Atomistix a/s. 2013. Available online: www.quantumwise.com (accessed on 15 June 2015).

45. Serra, S.; Tosatti, E.; Iarlori, S.; Scandolo, S.; Santoro, G. Interchain electron states in polyethylene. Phys. Rev. B 2000, 62, 4389-4393. [CrossRef]

(C) 2016 by the authors; licensee MDPI, Basel, Switzerland. This article is an open access article distributed under the terms and conditions of the Creative Commons Attribution (CC-BY) license (http://creativecommons.org/licenses/by/4.0/). 